\title{
Computed Tomographic Morphometry of Lumbar Spine in Indian Population
}

\author{
1Department of Radiodiagnosis, Sanjay Gandhi Postgraduate \\ Institute of Medical Sciences, Lucknow, Uttar Pradesh, India \\ ${ }^{2}$ Department of Neurosurgery, Sanjay Gandhi Postgraduate \\ Institute of Medical Sciences, Lucknow, Uttar Pradesh, India \\ ${ }^{3}$ Department of Biostatistics, Sanjay Gandhi Postgraduate Institute \\ of Medical Sciences, Lucknow, Uttar Pradesh, India \\ Indian J Neurotrauma 2022;19:12-18.
}

Vivek Singh ${ }^{1}$ Surya Nandan Prasad ${ }^{1, \odot}$ Zafar Neyaz ${ }^{1} \quad$ Nishant Bhargava ${ }^{1} \quad$ Uttam Yadav ${ }^{1}$

Arun Kumar Srivastav² Prabhakar Mishra $^{3} \quad$ Rajendra Vishnu Phadke ${ }^{1}$

\begin{abstract}
Address for correspondence Surya Nandan Prasad, MD, Department of Radiodiagnosis, Sanjay Gandhi Postgraduate Institute of Medical Sciences, Lucknow, Uttar Pradesh, India (e-mail: drsurya1@gmail.com).
\end{abstract}

\begin{abstract}
Keywords

- CT morphometry

- lumbar spine

- pedicle width

- vertebral height

- interpedicular distance

Purpose Several studies have been conducted to determine morphometry of lumbar vertebrae, mostly in western population and data on other populations is relatively sparse. Most of these studies have been carried out using fresh cadavers or osteological collections and several of them having limitations such as a small sample size and lack of demographic information. We conducted morphometric analysis of the lumbar vertebrae in a relatively large number of Indian patients using computed tomography scan. Vertebral body and pedicle dimensions of lumbar spinal elements were documented in Indian population and compared with other studies from the subcontinent as well as from other parts of the world. The morphometric data thus compiled may help in the development of new spinal implants for transpedicular screw fixations.

Methods An observational study was conducted and a total of 302 patients were evaluated. Thin section computed tomographic images of the 12th thoracic vertebra (D12) to 1st sacral vertebra (S1) were acquired and various dimensions of vertebral body and pedicle were recorded and analyzed.

Results Generally, the lumbar vertebral and pedicle dimensions were found to be greater in male patients. Comparison of dimensions in different populations revealed statistically significant differences in pedicle dimensions between Indian population and others.

Conclusion Morphometric analysis of lumbar vertebrae using computed tomography scan provides measurements of different vertebral dimensions. This will help in development of spinal implants in future to meet the specific needs of the studied population.
\end{abstract}

published online April 15, 2021
DOI https://doi.org/

10.1055/s-0041-1725568

ISSN 0973-0508. (c) 2021. Neurotrauma Society of India.

This is an open access article published by Thieme under the terms of the Creative Commons Attribution-NonDerivative-NonCommercial-License, permitting copying and reproduction so long as the original work is given appropriate credit. Contents may not be used for commercial purposes, or adapted, remixed, transformed or built upon. (https://creativecommons.org/licenses/by-nc-nd/4.0/).

Thieme Medical and Scientific Publishers Pvt. Ltd. A-12, 2nd Floor, Sector 2, Noida-201301 UP, India 


\section{Introduction}

Vertebral column forms central axis of the body and it gives main support to bones and muscles. ${ }^{1}$ Knowledge of vertebral morphology helps us to understand spine biomechanics. Thorough knowledge of lumbar morphometry is very helpful in correcting deformities and stabilizing the spine with the help of different spinal implants during spinal surgeries or in the setting of trauma. ${ }^{2,3}$ To ensure precise screw positioning, it is essential that the surgeons have an intimate knowledge of spinal anatomy and be able to localize the bony and neural structures accurately while performing the procedure. ${ }^{4,5}$

In different spinal ailments including trauma transpedicular fixation of the spine is an effective vertebral stabilization method. It is important to achieve proper placement of pedicle screw for a successful outcome in the spinal surgeries. ${ }^{6-10}$ Mismatching of pedicle and screw size may result in serious complications like loosening of the screw, fracture of the pedicle, dural tear, leakage of cerebrospinal fluid, nerve-root injuries. and in worst cases injury to spinal cord itself., ${ }^{611-13}$ Thus, detailed understanding of morphometry of the pedicle is important to prevent potential complications due to use of inappropriate sized pedicle screws.

Several studies have been conducted to determine morphometry of lumbar vertebrae, mostly in western population. Most of these studies have been carried out using fresh cadaver or osteological collections. Moreover, many of these studies have limitations such as a small sample size and lack of demographic data including race, age, and sex. ${ }^{14-17}$ Computed tomographic images have been employed more recently to study lumbar vertebral anatomy. ${ }^{18-20}$ Recent use of computed tomography (CT) for measurement of different vertebral dimensions like pedicle diameter, interpedicular distance, transpedicular angle, and other vertebral dimension has led to better evaluation of vertebral morphometry compared to plain X-rays and cadaveric studies.

In this study, we have conducted morphometric analysis of the lumbar vertebrae in Indian population in a relatively large number of patients by using CT scan. The morphometric data thus compiled may serve as a basis for the development of new spinal implants for transpedicular screw fixations.

\section{Materials and Methods}

This observational study was conducted in one of North India's premier tertiary care institute and this research had been approved by the institutional review board of the authors' affiliated institutions. A total of 302 patients undergoing CT evaluation in the radiology department were evaluated over a period of 1 year. Patients undergoing diagnostic CT scan for abdominal or genitourinary complaints and patients attending the radiology department for an abdominal CT scan for indications other than the vertebral column pathology during the study period were included in the study. Exclusion criteria included all individuals with age $<20$ years or age $>40$ years, cases with gross spinal pathology and neurological deficit due to spinal condition, major chronic systemic disease like chronic liver disease and chronic kidney disease, cases of clinically labeled dwarfism, and scans with motion/metallic artifacts.

CT scan was performed using 64-slice multidetector CT scan (Brilliance 190P 64-channel CT scanner, Philips). Noncontrast CT was performed from the level of diaphragm to pubic symphysis with area to be covered (field of view) from D12 vertebral level to the S1 vertebral level. The scan was acquired with slice thickness of $3 \mathrm{~mm}$ and then $1 \mathrm{~mm}$ thick images were reconstructed in bone window setting from the source data. Then the images were reformatted in axial, sagittal, and coronal planes and analyzed.

In our study, we measured different parameters of each vertebra from D12 to S1 level. For simplicity, we divided these parameters broadly into two groups:

1. Pedicle dimensions (-Fig. 1A, 1B and 1D):

Pedicle width-distance between medial and lateral surface of pedicle at midpoint.

Pedicle height-distance between superior and inferior border of pedicle at midpoint.

Interpedicular distance-maximum distance between pedicles.

Transpedicular angle-angle between a line passing through the center of the pedicle in longitudinal axis and a line parallel to the vertebral body midline.

CT ratio-pedicle transverse diameter/mean vertebral body width, expressed in percentage.

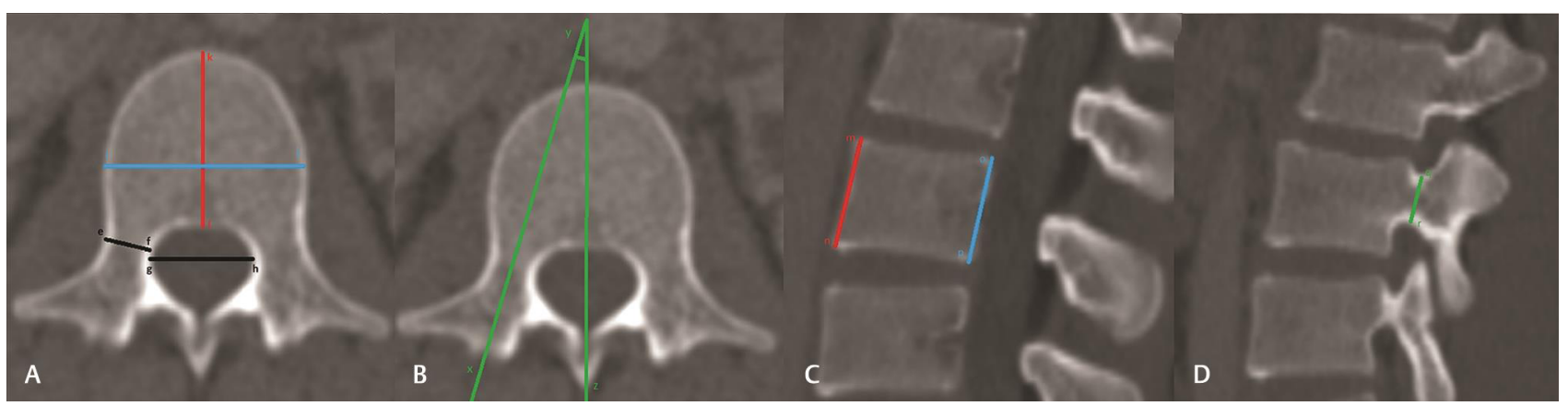

Fig. 1 Computed tomography (CT) lumbar vertebra in axial (A, B), mid-sagittal (C), and parasagittal (D) planes depicting pedicle width (E, F), interpedicular distance $(\mathbf{G}, \mathbf{H})$, vertebral width $(\mathbf{I}, \mathbf{J})$, vertebral depth $(\mathbf{K}, \mathbf{L})$, transpedicular angle $(x-y-z)$, anterior $(\mathbf{M}, \mathbf{N})$ and posterior $(\mathbf{O}, \mathbf{P})$ vertebral height, and pedicle height $(\mathbf{Q}, \mathbf{R})$. 
2. Vertebral body dimensions ( $\mathbf{- F i g .} \mathbf{1 A}$ and $\mathbf{1 C}$ ):

Upper and lower vertebral depth-distance between anterior and posterior surface of vertebral body in the plane of the upper and lower endplates, respectively.

Upper and lower vertebral width-distance between the lateral borders of the vertebral body in the plane of the upper and lower endplates, respectively.

Anterior vertebral body height-distance between the upper and lower endplates of vertebral body at anterior margin.

Posterior vertebral body height-the distance between the upper and lower endplates of vertebral body at posterior margin.

Data was processed and analyzed on SPSS version 17 (SPSS Inc.; Chicago, Illinois, United States), with $p$-value of 0.05 set to be significant. "Unpaired $t$-test" was used to compare different dimensions of lumbar spine of Indian patients with other population and "independent $t$-test" was used to compare between male and female population.

\section{Results}

Out of 302 patients studied, 174 (57.6\%) were male and 128 (42.4\%) were female. Note that $176(58.3 \%)$ of them were between the age of 20 and 30 years and 126 (41.7\%) were between the age of 31 and 40 years.

\section{Pedicle dimensions ( $\mathbf{- T a b l e ~} \mathbf{1}$ ):}

Mean pedicle width consistently increased from L1 to L5 in both males and females. The maximum mean pedicle width was at L5 and minimum at the L1 level. The pedicle wiOdth was significantly $(p<0.05)$ greater in males compared to females. Pedicle height decreased marginally from D12 to L4 followed by increase at the L5 level in both males and females. The minimum pedicle height was noted at L4 and maximum at D12 level. The pedicle height was significantly $(p<0.05)$ more in males compared to females at all levels.
Interpedicular distance constantly increased from D12 to L5 level in both males and females. The minimum interpedicular distance was noted at D12 and maximum at L5 level. The interpedicular distance was significantly $(p<0.05)$ more in males compared to females. The transpedicular angle decreased from D12 to L2 level followed by increase at L3 and then gradually increased from L3 to L5 level in both males and females. Maximum transpedicular angle was noted at L5 and minimum at L2 level. There was insignificant $(p$-value $>0.05$ ) difference present in the transpedicular angle between males and females.

CT ratio decreased marginally from D12 to L2 followed by increase from L3 to L5 level. The maximum CT ratio was recorded at D12 and minimum at L2 in both males and females. There was no significant difference between males and females; however, significant difference between right and left was seen at all levels.

2. Dimensions of vertebral body ( - Table 2 ):

Upper vertebral depth progressively increased from D12 to L5 level, then it decreased at S1 in both males and females. The minimum upper vertebral depth was at D12 and maximum at L5. Upper vertebral depth was significantly $(p<0.05)$ larger in males compared to females at all levels. Lower vertebral depth increased from D12 to L4 followed by decrease from L5 to S1 level in males but in females it progressively increased from D12 to L5 followed by decrease at the $\mathrm{S} 1$ level. The maximum lower vertebral depth was seen at L4 level in males and at L5 level in females, whereas minimum lower vertebral depth was noted at $\mathrm{S} 1$ level in both males and females. Lower vertebral depth was significantly $(p<0.05)$ more in males at all level.

Upper vertebral width progressively increased from D12 to S1 level in both males and females. The minimum upper vertebral width was at D12 and maximum at S1 level. Upper vertebral width was significantly $(p<0.05)$ larger in males compared to females at all levels. Lower vertebral width increased from D12 to L5 but decreased at S1 level in both males and females. Lower vertebral

Table 1 Measurements (in $\mathrm{mL}$ ) of mean \pm SD of pedicles width, pedicles height, interpedicular distance, and transpedicular angle (in degree) in males (M) and females (F)

\begin{tabular}{|c|c|c|c|c|c|c|c|c|c|c|c|c|}
\hline & \multicolumn{2}{|l|}{ D12 } & \multicolumn{2}{|l|}{ L1 } & \multicolumn{2}{|l|}{ L2 } & \multicolumn{2}{|l|}{ L3 } & \multicolumn{2}{|l|}{ L4 } & \multicolumn{2}{|l|}{ L5 } \\
\hline & $M$ & $F$ & $M$ & $F$ & $M$ & $F$ & $M$ & F & $M$ & $F$ & $M$ & $F$ \\
\hline $\begin{array}{l}\text { Pedicle width } \\
\text { (right) }\end{array}$ & $\begin{array}{l}11.4 \pm \\
1.72\end{array}$ & $\begin{array}{l}10.57 \pm \\
1.97\end{array}$ & $\begin{array}{l}9.08 \pm \\
1.60\end{array}$ & & $\begin{array}{l}9.60 \pm \\
1.43\end{array}$ & & $\begin{array}{l}10.81 \pm \\
1.69\end{array}$ & $\begin{array}{l}10.01 \pm \\
1.47\end{array}$ & $\begin{array}{l}11.85 \pm \\
1.75\end{array}$ & $\begin{array}{l}11.14 \pm \\
1.58\end{array}$ & $\begin{array}{l}14.43 \pm \\
2.24\end{array}$ & $\begin{array}{l}13.48 \pm \\
2.29\end{array}$ \\
\hline $\begin{array}{l}\text { Pedicle width } \\
\text { (left) }\end{array}$ & $\begin{array}{l}11.69 \pm \\
1.74\end{array}$ & $\begin{array}{l}10.77 \pm \\
1.95\end{array}$ & $\begin{array}{l}9.79 \pm \\
1.61\end{array}$ & & \begin{tabular}{|l|}
$10.46 \pm$ \\
1.61
\end{tabular} & & $\begin{array}{l}11.49 \pm \\
1.73\end{array}$ & $\begin{array}{l}10.54 \pm \\
1.68\end{array}$ & \begin{tabular}{|l}
$12.63 \pm$ \\
1.97
\end{tabular} & \begin{tabular}{|l|}
$11.77 \pm$ \\
1.59
\end{tabular} & $\begin{array}{l}14.78 \pm \\
2.05\end{array}$ & $\begin{array}{l}13.84 \pm \\
1.97\end{array}$ \\
\hline Pedi & $\begin{array}{l}14.6 \pm \\
1.86\end{array}$ & & $\begin{array}{l}14.44 \pm \\
1.85\end{array}$ & $\begin{array}{l}13.77 \pm \\
1.64\end{array}$ & $\begin{array}{l}14.25 \pm \\
1.75\end{array}$ & $\begin{array}{l}13.25 \pm \\
1.71\end{array}$ & \begin{tabular}{|l}
$14.15 \pm$ \\
1.81
\end{tabular} & $\begin{array}{l}13.21 \pm \\
1.75\end{array}$ & $\begin{array}{l}13.11 \pm \\
1.80\end{array}$ & $\begin{array}{l}12.14 \pm \\
1.88\end{array}$ & $\begin{array}{l}13.44 \pm \\
1.75\end{array}$ & $\begin{array}{l}12.53 \pm \\
1.83\end{array}$ \\
\hline $\begin{array}{l}\text { Interpedi } \\
\text { distance }\end{array}$ & $\begin{array}{l}20.97 \pm \\
2.36\end{array}$ & $\begin{array}{l}20.17 \pm \\
2.03\end{array}$ & $\begin{array}{l}21.64 \pm \\
2.22\end{array}$ & $\begin{array}{l}20.65 \pm \\
2.00\end{array}$ & $\begin{array}{l}22.57 \pm \\
2.79\end{array}$ & $\begin{array}{l}21.36 \pm \\
2.26\end{array}$ & $\begin{array}{l}24.70 \pm \\
2.42\end{array}$ & \begin{tabular}{|l}
$23.34 \pm$ \\
2.37
\end{tabular} & $\begin{array}{l}27.65 \pm \\
3.24\end{array}$ & $\begin{array}{l}26.93 \pm \\
3.31\end{array}$ & $\begin{array}{l}34.02 \pm \\
4.37\end{array}$ & $\begin{array}{l}32.47 \pm \\
4.35\end{array}$ \\
\hline $\begin{array}{l}\text { Transpedicular } \\
\text { angle (right) }\end{array}$ & $\begin{array}{l}30.85 \pm \\
4.66\end{array}$ & $\begin{array}{l}31.35 \pm \\
4.22\end{array}$ & $\begin{array}{l}26.43 \pm \\
3.26\end{array}$ & $\begin{array}{l}26.55 \pm \\
2.95\end{array}$ & $\begin{array}{l}26.15 \pm \\
3.57\end{array}$ & $\begin{array}{l}26.61 \pm \\
3.11\end{array}$ & $\begin{array}{l}28.10 \pm \\
3.81\end{array}$ & $\begin{array}{l}27.89 \pm \\
3.42\end{array}$ & $\begin{array}{l}30.83 \pm \\
4.36\end{array}$ & $\begin{array}{l}31.17 \pm \\
3.96\end{array}$ & $\begin{array}{l}40.30 \pm \\
8.59\end{array}$ & $\begin{array}{l}40.44 \pm \\
8.42\end{array}$ \\
\hline $\begin{array}{l}\text { Transpedicular } \\
\text { angle (left) }\end{array}$ & $\begin{array}{l}32.47 \pm \\
4.34\end{array}$ & $\begin{array}{l}33.95 \pm \\
6.36\end{array}$ & $\begin{array}{l}28.43 \pm \\
3.45\end{array}$ & $\begin{array}{l}28.85 \pm \\
4.39\end{array}$ & $\begin{array}{l}28.20 \pm \\
3.42\end{array}$ & $\begin{array}{l}28.66 \pm \\
3.22\end{array}$ & $\begin{array}{l}29.36 \pm \\
3.69\end{array}$ & $\begin{array}{l}29.78 \pm \\
3.58\end{array}$ & $\begin{array}{l}31.71 \pm \\
4.49\end{array}$ & $\begin{array}{l}32.28 \pm \\
4.12\end{array}$ & $\begin{array}{l}39.17 \pm \\
7.77\end{array}$ & $\begin{array}{l}40.23 \pm \\
8.40\end{array}$ \\
\hline
\end{tabular}

Abbreviation: SD, standard deviation. 
Table 2 Measurements (in $\mathrm{mL}$ ) of mean \pm SD vertebral body dimensions in males (M) and females (F)

\begin{tabular}{|c|c|c|c|c|c|c|c|c|c|c|c|c|c|c|}
\hline & \multicolumn{2}{|l|}{ D12 } & \multicolumn{2}{|l|}{ L1 } & \multicolumn{2}{|l|}{ L2 } & \multicolumn{2}{|l|}{ L3 } & \multicolumn{2}{|l|}{ L4 } & \multicolumn{2}{|l|}{ L5 } & \multicolumn{2}{|l|}{ S1 } \\
\hline & $M$ & $F$ & $M$ & $F$ & $M$ & $F$ & $M$ & $F$ & $M$ & $F$ & $M$ & $F$ & $M$ & $F$ \\
\hline $\begin{array}{l}\text { Upper } \\
\text { vertebral } \\
\text { depth }\end{array}$ & $\begin{array}{l}27.09 \pm \\
2.59\end{array}$ & $\begin{array}{l}25.10 \pm \\
2.46\end{array}$ & $\begin{array}{l}28.61 \pm \\
2.61\end{array}$ & $\begin{array}{l}26.53 \pm \\
2.22\end{array}$ & $\begin{array}{l}30.37 \pm \\
3.26\end{array}$ & $\begin{array}{l}28.30 \pm \\
2.36\end{array}$ & $\begin{array}{l}31.37 \pm \\
2.78\end{array}$ & $\begin{array}{l}29.77 \pm \\
2.87\end{array}$ & $\begin{array}{l}31.56 \pm \\
2.27\end{array}$ & $\begin{array}{l}30.12 \pm \\
2.22\end{array}$ & $\begin{array}{l}32.41 \pm \\
2.35\end{array}$ & $\begin{array}{l}30.94 \pm \\
2.41\end{array}$ & $\begin{array}{l}25.10 \pm \\
3.19\end{array}$ & $\begin{array}{l}23.47 \pm \\
3.09\end{array}$ \\
\hline $\begin{array}{l}\text { Lower } \\
\text { vertebral } \\
\text { depth }\end{array}$ & $\begin{array}{l}27.88 \pm \\
2.53\end{array}$ & $\begin{array}{l}25.82 \pm \\
2.73\end{array}$ & $\begin{array}{l}29.49 \pm \\
2.90\end{array}$ & $\begin{array}{l}27.31 \pm \\
3.24\end{array}$ & $\begin{array}{l}30.60 \pm \\
2.60\end{array}$ & $\begin{array}{l}29.00 \pm \\
2.39\end{array}$ & $\begin{array}{l}31.25 \pm \\
2.39\end{array}$ & $\begin{array}{l}29.58 \pm \\
2.28\end{array}$ & $\begin{array}{l}32.15 \pm \\
3.04\end{array}$ & $\begin{array}{l}30.00 \pm \\
3.29\end{array}$ & $\begin{array}{l}31.66 \pm \\
2.89\end{array}$ & $\begin{array}{l}30.30 \pm \\
2.52\end{array}$ & $\begin{array}{l}22.78 \pm \\
2.66\end{array}$ & $\begin{array}{l}22.03 \pm \\
2.98\end{array}$ \\
\hline $\begin{array}{l}\text { Upper } \\
\text { vertebral } \\
\text { width }\end{array}$ & $\begin{array}{l}36.99 \pm \\
2.89\end{array}$ & $\begin{array}{l}34.62 \pm \\
2.82\end{array}$ & $\begin{array}{l}40.15 \pm \\
3.09\end{array}$ & $\begin{array}{l}36.95 \pm \\
3.14\end{array}$ & $\begin{array}{l}42.55 \pm \\
4.08\end{array}$ & $\begin{array}{l}39.49 \pm \\
2.97\end{array}$ & $\begin{array}{l}44.34 \pm \\
3.17\end{array}$ & $\begin{array}{l}41.54 \pm \\
3.47\end{array}$ & $\begin{array}{l}46.42 \pm \\
3.98\end{array}$ & $\begin{array}{l}43.59 \pm \\
3.58\end{array}$ & $\begin{array}{l}48.79 \pm \\
5.54\end{array}$ & $\begin{array}{l}46.00 \pm \\
3.66\end{array}$ & $\begin{array}{l}49.08 \pm \\
4.40\end{array}$ & $\begin{array}{l}46.17 \pm \\
4.74\end{array}$ \\
\hline $\begin{array}{l}\text { Lower } \\
\text { vertebral } \\
\text { width }\end{array}$ & $\begin{array}{l}39.30 \pm \\
3.16\end{array}$ & $\begin{array}{l}36.73 \pm \\
2.99\end{array}$ & $\begin{array}{l}42.26 \pm \\
4.50\end{array}$ & $\begin{array}{l}39.85 \pm \\
3.13\end{array}$ & $\begin{array}{l}44.35 \pm \\
3.27\end{array}$ & $\begin{array}{l}42.07 \pm \\
5.53\end{array}$ & $\begin{array}{l}46.66 \pm \\
3.38\end{array}$ & $\begin{array}{l}43.98 \pm \\
3.44\end{array}$ & $\begin{array}{l}46.86 \pm \\
3.85\end{array}$ & $\begin{array}{l}44.92 \pm \\
3.32\end{array}$ & $\begin{array}{l}46.93 \pm \\
3.36\end{array}$ & $\begin{array}{l}45.91 \pm \\
3.39\end{array}$ & $\begin{array}{l}30.78 \pm \\
3.45\end{array}$ & $\begin{array}{l}29.67 \pm \\
4.46\end{array}$ \\
\hline $\begin{array}{l}\text { Anterior } \\
\text { vertebral } \\
\text { height }\end{array}$ & $\begin{array}{l}22.59 \pm \\
2.18\end{array}$ & \begin{tabular}{|l|}
$21.85 \pm$ \\
1.78
\end{tabular} & $\begin{array}{l}24.13 \pm \\
1.88\end{array}$ & $\begin{array}{l}23.28 \pm \\
1.74\end{array}$ & $\begin{array}{l}25.53 \pm \\
1.96\end{array}$ & $\begin{array}{l}24.58 \pm \\
2.79\end{array}$ & $\begin{array}{l}26.09 \pm \\
2.10\end{array}$ & $\begin{array}{l}24.90 \pm \\
2.05\end{array}$ & $\begin{array}{l}25.72 \pm \\
2.01\end{array}$ & \begin{tabular}{|l|}
$24.77 \pm$ \\
2.30
\end{tabular} & $\begin{array}{l}26.35 \pm \\
2.27\end{array}$ & $\begin{array}{l}25.22 \pm \\
2.73\end{array}$ & $\begin{array}{l}30.14 \pm \\
2.93\end{array}$ & $\begin{array}{l}28.78 \pm \\
3.05\end{array}$ \\
\hline $\begin{array}{l}\text { Posterior } \\
\text { vertebral } \\
\text { height }\end{array}$ & $\begin{array}{l}24.34 \pm \\
2.13\end{array}$ & $\begin{array}{l}23.06 \pm \\
1.89\end{array}$ & $\begin{array}{l}25.55 \pm \\
2.66\end{array}$ & $\begin{array}{l}24.36 \pm \\
2.67\end{array}$ & $\begin{array}{l}26.50 \pm \\
2.03\end{array}$ & $\begin{array}{l}24.90 \pm \\
3.32\end{array}$ & $\begin{array}{l}26.83 \pm \\
1.90\end{array}$ & $\begin{array}{l}24.34 \pm \\
2.16\end{array}$ & $\begin{array}{l}24.49 \pm \\
2.09\end{array}$ & $\begin{array}{l}22.34 \pm \\
3.26\end{array}$ & $\begin{array}{l}22.62 \pm \\
2.00\end{array}$ & $\begin{array}{l}21.34 \pm \\
2.90\end{array}$ & $\begin{array}{l}22.40 \pm \\
2.84\end{array}$ & $\begin{array}{l}20.69 \pm \\
2.74\end{array}$ \\
\hline
\end{tabular}

Abbreviation: SD, standard deviation.

width was significantly $(p<0.05)$ larger in males at all level. Anterior vertebral body height gradually increased from D12 to L3 but marginally decreased at L4 followed by increase at L5 and S1 level in both males and females. Anterior vertebral body height was significantly $(p<0.05)$ greater in males at all levels. Posterior vertebral body height gradually increased from D12 to L3 level followed by decrease from L4 to S1 level in both males and females. Posterior vertebral body height was significantly $(p<0.05)$ more in males at all levels.

\section{Discussion}

There are multiple studies in the literature on the anatomic and radiographic morphometry of the lumbar spine. However, the values vary significantly across races, genders, and study groups. Majority of studies available have been performed in non-Asian population. Very limited data regarding the anatomic detail of lumbar spine is available among Asian population. ${ }^{21-25}$ On review of literature there are multiple studies on Indian population of different regions; however, several of these studies have limitations such as small sample size and lack of information on demographic data like race, age, and sex. ${ }^{24-30}$ Differences have been reported between Indian and the western population by Chadha. et al and Acharya. et al on the different dimension of lumbar vertebrae, but these studies did not mention the differences between male and female vertebral parameters and also these studies were conducted on relatively small sample size. ${ }^{24,25}$ This necessitated the need for morphometric analysis of the lumbar spine among Indian population. Our study compiled morphometric data of lower thoracic and lumbar vertebrae (from D12 to S1 vertebra) in an Indian population by CT-based measurements which were more clinically relevant and accurate in contrast to cadaveric or manually measured data. The present study provides a comparison with other
Indian, Asian, as well as western population and also provides comparison between males and females. The morphometry of lumbar vertebrae thus obtained will provide necessary parameters for developing accurate size spinal implants and pedicle screws for Indian population in future.

1. Pedicle dimension:

In our study, we noted that mean pedicle width increased gradually from L1 to L5 level with maximum mean pedicle width at L5 and minimum at L1 level. The pedicle width diameters were smaller in female at all levels. Various other studies showed similar trends but ours is the largest series and compared both males and females of Indian population ( - Table 3). Multiple international studies from Asian countries which showed similar trends include one of Alam et al, where they conducted CT-based measurements of lumbar vertebrae on 49 patients in Pakistan. ${ }^{22}$

The pedicle height progressively decreased from D12 to L4 then increased at L5 both in males and females and the pedicle height was greater in males. Various studies showed similar results like by Sreevidya et al and Singh et al, but these were cadaveric studies. ${ }^{15,26}$ Few studies like that of Arora et al and Seema. et al found that the pedicle height gradually increased from L1 to L5 level., ${ }^{6,10}$ This was in contradiction to our study. Alam. et al in Pakistani population showed pedicle height decreased from L1 to L5 level. ${ }^{22}$ Wolf. et al in Israeli population showed that the pedicle height decreased from L1 to L3 then increased at L4 and L5 level. ${ }^{21}$ This pattern was slightly different from our study. Interpedicular distance increased from D12 to L5 level in both males and females. Similar patterns were seen in various studies done in other populations, however, absolute measurements differed ( - Table 4 ).

CT ratio (pedicle transverse diameter/vertebral body transverse diameter, expressed in percentage) decreased marginally from D12 to L2 followed by increase from L3 to 
Table 3 Pedicle width comparison with other Indian and Asian studies

\begin{tabular}{|c|c|c|c|c|c|}
\hline Our study & L1 & L2 & L3 & L4 & L5 \\
\hline Male $(n=174)$ & $9.43 \pm 1.60$ & $10.03 \pm 1.43$ & $11.19 \pm 1.69$ & $12.24 \pm 1.75$ & $14.60 \pm 2.24$ \\
\hline Female $(n=128)$ & $8.72 \pm 1.45$ & $9.07 \pm 1.41$ & $10.27 \pm 1.47$ & $11.45 \pm 1.58$ & $13.66 \pm 2.29$ \\
\hline \multicolumn{6}{|l|}{ Other Indian studies } \\
\hline Chadha. et al $(n=31)$ & $6.69 \pm 1.55$ & $7.26 \pm 1.43$ & $8.43 \pm 1.42$ & $10.81 \pm 1.17$ & $13.47 \pm 1.78$ \\
\hline Acharya. et al $(n=50)$ & $7.2 \pm 0.93$ & $7.62 \pm 0.84$ & $8.97 \pm 1.09$ & $11.12 \pm 1.01$ & $13.91 \pm 1.16$ \\
\hline Rajput. et al $(n=25)$ & $7.24 \pm 2.22$ & $7.86 \pm 2.66$ & $9.11 \pm 2.79$ & $10.45 \pm 2.35$ & $12 \pm 4.39$ \\
\hline Kumar. et al $(n=61)$ & $8.845 \pm 1.90$ & $9.05 \pm 1.94$ & $10.85 \pm 1.62$ & $12.6 \pm 1.78$ & $17.1 \pm 2.40$ \\
\hline Mitra. et al $(n=20)$ & 7.5 & 7.59 & 8.65 & 9.81 & 14.64 \\
\hline Singh. et al $(n=20)$ & $7.5 \pm 1.54$ & $7.8 \pm 1.75$ & $9.7 \pm 2.03$ & $12.1 \pm 2.24$ & $17.1 \pm 2.9$ \\
\hline $\begin{array}{l}\text { Sreevidya. et al (South Indian } \\
\text { population, } n=20 \text { ) }\end{array}$ & $8.2 \pm 2.9$ & $8 \pm 1.7$ & $8.5 \pm 1.8$ & $10.2 \pm 1.4$ & $13.1 \pm 2.5$ \\
\hline $\begin{array}{l}\text { Arora. et al (Delhi population, } \\
n=26 \text { ) }\end{array}$ & 7.51 & 7.95 & 8.75 & 13 & 15.34 \\
\hline $\begin{array}{l}\text { Singel. et al (Saurashtra } \\
\text { population, } n=60 \text { ) }\end{array}$ & $8.2 \pm 6.7$ & $8.5 \pm 6.5$ & $10.4 \pm 7.0$ & $13.5 \pm 7.0$ & $13.5 \pm 9.7$ \\
\hline \multicolumn{6}{|l|}{ Other Asian studies } \\
\hline $\begin{array}{l}\text { Marasini. et al (Nepalese } \\
\text { population, } n=246 \text { ) }\end{array}$ & 7.17 & 7.62 & 9.5 & 10.57 & 11.3 \\
\hline $\begin{array}{l}\text { Wolf et al } \\
\text { (Israeli population, } n=55 \text { ) }\end{array}$ & $5.6 \pm 1.3$ & $7.7 \pm 1.5$ & $8.9 \pm 1.9$ & $11.4 \pm 1.8$ & $13.7 \pm 2.2$ \\
\hline $\begin{array}{l}\text { Alam. et al (Pakistani } \\
\text { population, } n=49 \text { ) }\end{array}$ & 6.25 & 7.29 & 10.59 & 10.59 & 13.53 \\
\hline $\begin{array}{l}\text { Maaly. et al (Egyptian } \\
\text { population, } n=76 \text { ) }\end{array}$ & $7.7 \pm 1.6$ & $8.7 \pm 1.4$ & $10.6 \pm 1.6$ & $13.6 \pm 1.9$ & $18.3 \pm 2.5$ \\
\hline $\begin{array}{l}\text { Kang et al } \\
\text { (Korean population, } n=50 \text { ) }\end{array}$ & $8.61 \pm 1.70$ & $8.95 \pm 1.50$ & $10.63 \pm 1.40$ & $12.14 \pm 2.01$ & $17.08 \pm 2.40$ \\
\hline
\end{tabular}

Table 4 Interpedicular distance comparison with other studies

\begin{tabular}{|c|c|c|c|c|c|}
\hline & L1 & $\mathrm{L} 2$ & L3 & L4 & L5 \\
\hline \multicolumn{6}{|l|}{ Our study ( $n=302$ ) } \\
\hline Male & $21.64 \pm 2.22$ & $22.57 \pm 2.79$ & $24.70 \pm 2.42$ & $27.65 \pm 3.24$ & $34.02 \pm 4.37$ \\
\hline Female & $20.65 \pm 2.00$ & $21.36 \pm 2.26$ & $23.34 \pm 2.37$ & $26.93 \pm 3.31$ & $32.47 \pm 4.35$ \\
\hline \multicolumn{6}{|l|}{ Mitra. et al $(n=20)$} \\
\hline Male & 37.3 & 38.5 & 38 & 37.1 & \\
\hline Female & 37.5 & 38 & 40 & 37 & \\
\hline Seema. et al $(n=100)$ & $23.95 \pm 0.30$ & $24.96 \pm 0.40$ & $26.64 \pm 0.32$ & $27.89 \pm 0.41$ & $30.93 \pm 0.28$ \\
\hline Marasini. et al $(n=246)$ & 25.54 & 27.03 & 27.7 & 28.62 & 31.39 \\
\hline \multicolumn{6}{|l|}{ Alam. et al $(n=49)$} \\
\hline Male & 24.2 & 24.34 & 24.13 & 24.48 & 28.43 \\
\hline Female & 23.5 & 23.46 & 22.36 & 23.81 & 25.96 \\
\hline
\end{tabular}

L5 level. The maximum value was noted at D12 and minimum at L2 level (- Table 5). Till now "CT ratio" as a parameter was only documented in Korean population by Kang et al, where they used maximum transverse diameter of vertebral body as the denominator. ${ }^{23}$ They measured CT-based dimensions of lumbar vertebrae of 50 patients and showed the maximum CT ratio at L5 (31.13\%) and minimum at L2 (20.46\%). The authors in the study on Korean population observed that there was definite correlation between vertebral width and pedicle width measured by CT at specific vertebral level. Vertebral width can be measured with reasonable accuracy on plane radiograph taken in true anteroposterior (AP) projection. However, since pedicles are obliquely placed structures, it is not possible to know its accurate dimensions on AP or lateral radiographs. Thus, by knowing the $\mathrm{CT}$ ratio beforehand at specific vertebral levels 
and vertebral width measured on AP radiographs, one can very well estimate the pedicle width at that vertebral level. In our study, we used mean of upper and lower vertebral body width as the denominator and measured the "CT ratio" in the Indian population. Similar pattern was seen in our study; however, our values were higher than those documented in Korean population.

2. Vertebral dimension:

Anterior vertebral body height gradually increased from D12 to L3 level, marginally decreased at L4 followed by an increase at L5 and S1 level in both males and females. Similar results were recorded by Singh. et al in 20 cadavers in North Indian population. ${ }^{26}$ Wolf et $\mathrm{al}^{21}$ in his study observed that anterior vertebral body height gradually increased from L1 to L5 which was different from our results (-Table $\mathbf{6}$ ). Alam. et al showed that the anterior vertebral body height increased from L1 to L3 in male and female population of Pakistan as depicted in our study. ${ }^{22}$ Posterior vertebral body height gradually increased from D12 to L3 followed by progressive decrease from L3 to S1 level in both males and females ( - Table 7). Cadaver study on Indian patients by Singh. et al and CT morphometry in Pakistani population by Alam. et al mentioned similar pattern of posterior vertebral body height measurements in both males and females. ${ }^{22,26}$

Upper vertebral depth increased from D12 to L5 then it decreased at S1 in both males and females. Similar pattern was observed by Alam. et al in Pakistani population in both males and females. Also, the values of upper vertebral depth were greater in males compared to females at all levels similar to our study. ${ }^{22}$

Upper vertebral width increased from D12 to S1 in both males and females. Similar results were found by Singh. et al on 20 cadaveric study. ${ }^{26}$ Alam. et al in Pakistani population and Kang. et al in Korean population CT morphometry, observed similar trends of increase of upper vertebral width craniocaudally from L1 to L5.22,23 Lower vertebral width increased from D12 to L5 but decreased at S1 level

Table 5 Ct ratio (in \%) comparison with other studies

\begin{tabular}{|l|l|l|l|l|l|l|}
\hline \multirow{2}{*}{} & \multicolumn{5}{|c|}{ Our study $(n=302)$} & $\begin{array}{l}\text { Kang et al } \\
\text { (Korean population, } n=50)\end{array}$ \\
\cline { 2 - 6 } & Male $(n=174)$ & Female $(n=128)$ & Mean & Mean \\
\cline { 2 - 6 } & Right & Left & Right & Left & \\
\hline D12 & 29.89 & 30.64 & 29.62 & 30.18 & 30.09 & 23.31 \\
\hline L1 & 22.03 & 23.75 & 21.93 & 23.49 & 22.80 & 20.36 \\
\hline L2 & 22.09 & 24.07 & 21.11 & 23.37 & 22.66 & 20.46 \\
\hline L3 & 23.76 & 25.25 & 23.41 & 24.65 & 24.27 & 22.57 \\
\hline L4 & 25.41 & 27.08 & 25.17 & 26.59 & 26.07 & 24.71 \\
\hline L5 & 30.15 & 30.88 & 29.34 & 30.12 & 30.13 & 31.13 \\
\hline
\end{tabular}

Abbreviation: $\mathrm{CT}$, computed tomography.

Table 6 Anterior vertebral height comparison with other studies

\begin{tabular}{|l|l|l|l|l|l|l|}
\hline & $\begin{array}{l}\text { Our study } \\
\text { (Male, } \boldsymbol{n}=\mathbf{1 7 4})\end{array}$ & $\begin{array}{l}\text { Our study } \\
\text { (Female, } \boldsymbol{n}=\mathbf{1 2 8})\end{array}$ & $\begin{array}{l}\text { Singh et al } \\
(\boldsymbol{n}=\mathbf{2 0})\end{array}$ & $\begin{array}{l}\text { Wolf et al } \\
(\boldsymbol{n}=\mathbf{5 5})\end{array}$ & $\begin{array}{l}\text { Alam et al } \\
(\text { Male, } \boldsymbol{n}=\mathbf{3 3})\end{array}$ & $\begin{array}{l}\text { Alam et al } \\
(\text { Female, } \boldsymbol{n}=\mathbf{1 6})\end{array}$ \\
\hline L1 & $24.13 \pm 1.88$ & $23.28 \pm 1.74$ & $23.3 \pm 2.41$ & $24.9 \pm 2.4$ & 24.5 & 23.9 \\
\hline L2 & $25.53 \pm 1.96$ & $24.58 \pm 2.79$ & $24.7 \pm 2.31$ & $25.4 \pm 1.1$ & 26.65 & 25.61 \\
\hline L3 & $26.09 \pm 2.10$ & $24.9 \pm 2.05$ & $25.7 \pm 2.16$ & $25.6 \pm 1.6$ & 27.3 & 27.05 \\
\hline L4 & $25.72 \pm 2.01$ & $24.77 \pm 2.30$ & $25.4 \pm 2.39$ & $26.5 \pm 0.6$ & 27.46 & 26.92 \\
\hline L5 & $26.35 \pm 2.27$ & $25.22 \pm 2.73$ & $26.1 \pm 2.10$ & $28.6 \pm 1.3$ & 27.6 & 26.72 \\
\hline
\end{tabular}

Table 7 Posterior vertebral height comparison with other studies

\begin{tabular}{|l|l|l|l|l|l|}
\hline & $\begin{array}{l}\text { Our study } \\
(\text { Male, } \boldsymbol{n}=\mathbf{1 7 4})\end{array}$ & $\begin{array}{l}\text { Our study } \\
\text { (Female, } \boldsymbol{n}=\mathbf{1 2 8})\end{array}$ & $\begin{array}{l}\text { Singh et al } \\
(\boldsymbol{n}=\mathbf{2 0})\end{array}$ & $\begin{array}{l}\text { Alam et al } \\
(\text { Male, } \boldsymbol{n}=\mathbf{3 3})\end{array}$ & $\begin{array}{l}\text { Alam et al } \\
(\text { Female, } \boldsymbol{n}=16)\end{array}$ \\
\hline L1 & $25.55 \pm 2.66$ & $24.36 \pm 2.67$ & $26.4 \pm 1.77$ & 28.2 & 25.6 \\
\hline L2 & $26.5 \pm 2.03$ & $24.9 \pm 3.32$ & $26.5 \pm 2.35$ & 29.23 & 26.69 \\
\hline L3 & $26.83 \pm 1.90$ & $24.34 \pm 2.16$ & $26.5 \pm 2.03$ & 28.55 & 27.47 \\
\hline L4 & $24.49 \pm 2.09$ & $22.34 \pm 3.26$ & $25.5 \pm 2.22$ & 27.1 & 26.21 \\
\hline L5 & $22.62 \pm 2.00$ & $21.34 \pm 2.9$ & $23.1 \pm 4.72$ & 24.84 & 23.9 \\
\hline
\end{tabular}


in both males and females. Similar study by Singh. et al on 20 cadavers in North Indian population noted that lower vertebral width increased from L1 to L2 then decreased at L3 followed by an increase at L4 and L5 levels. ${ }^{26}$ This pattern was not seen in our study. The study of Alam. et al on Pakistani population showed similar patterns in both males and females as seen in our study with lower vertebral width greater in males. ${ }^{22}$ So our study results are very similar to Pakistani population study results for pedicle and vertebral body dimensions but not to other Asian population where statistically significant differences were noted.

\section{Conclusion}

The limited available data on the anatomical details of lumbar spine for large sample size of Indian and Asian population necessitated need for morphometric analysis of lumbar vertebrae among Indian patients. Computed tomographic image evaluation for lumbar morphometry is clinically more relevant and accurate than cadaveric or manually measured data. This study may provide necessary parameters for developing accurate size spinal implants and pedicle screws for Indian population in future.

\section{Funding \\ None.}

\section{Conflict of Interest}

None declared.

\section{References}

1 Jadhav AS, Katti AS, Herekar NG, Jadhav SB. Osteological study of lumbar vertebrae in Western Maharashtra population. J Anat Soc India 2013;62(1):10-16

2 Chandni G, Poonam K, Arvind KP, Antony SD. Morphometric study of pedicles in lumbar vertebrae and its clinical significance. J Surg Acad 2014;4(1):37-40

3 Olsewski JM, Simmons EH, Kallen FC, Mendel FC, Severin CM, Berens DL. Morphometry of the lumbar spine: anatomical perspectives related to transpedicular fixation. J Bone Joint Surg Am 1990;72(4):541-549

4 Lorenz MA, Hodges S, Vrbos LA. Spinal fixation. Curr Opin Orthop 1993;4(2):192-204

5 Zindrick MR. The role of transpedicular fixation systems for stabilization of the lumbar spine. Orthop Clin North Am 1991;22(2):333-344

6 Arora L, Dada R, Singh V. Morphometric study of lumbar pedicles in Delhi region of northern India. Indian J Practising Doctor 2005;3(5):11-12

7 Aruna N, Rajeshwari T. A study of lumbar pedicle size in South Indians. Anatomica Karnataka 2011;5(2):69-73

8 Bin L, Yong H, Yang X, Bi Z, Mo S, Zhi-Min G. Pedicle screw piercer with warning device - a technique to increase accuracy of pedicle screw placement: a cadaveric study. Indian J Orthop 2014;48(6):545-549

9 Mitra SR, Datir SP, Jadhav SO. Morphometric study of the lumbar pedicle in the Indian population as related to pedicular screw fixation. Spine 2002;27(5):453-459

10 Singh M. Morphometric study of pedicles of the lumbar vertebrae in adult Punjabi males. Int J Anat Res 2016;4(2):2401-2404

11 Zindrick MR, Wiltse LL, Widell EH, et al. A biomechanical study of intrapeduncular screw fixation in the lumbosacral spine. Clin Orthop Relat Res 1986;203(203):99-112
12 Esses SI, Sachs BL, Dreyzin V. Complications associated with the technique of pedicle screw fixation. A selected survey of ABS members. Spine 1993;18(15):2231-2238, discussion 2238-2239

13 Weinstein JN, Rydevik BL, Rauschning W. Anatomic and technical considerations of pedicle screw fixation. Clin Orthop Relat Res 1992;(284):34-46

14 Krag MH, Beynnon BD, Pope MH, Frymoyer JW, Haugh LD, Weaver DL. An internal fixator for posterior application to short segments of the thoracic, lumbar, or lumbosacral spine. Design and testing. Clin Orthop Relat Res 1986;(203):75-98

15 Sreevidya J, Dharani V, Savithri K, Seshayyan S. Study of lumbar vertebrae with respect to the dimensions of the pedicle in South Indian population. Indian J Basic Appl Med Res 2017;6(2):523-530

16 Misenhimer GR, Peek RD, Wiltse LL, Rothman SL, Widell EH Jr. Anatomic analysis of pedicle cortical and cancellous diameter as related to screw size. Spine 1989;14(4):367-372

17 Saillant G. Anatomical study of the vertebral pedicles. Surgical application [in French]. Rev Chir Orthop Repar Appar Mot 1976;62(2):151-160

18 Kumar AP, Kasturi A, Ramu C, Nadadur S. Morphometric analysis of lumbar vertebrae in tertiary care institutions in Telangana. Ann Int Med Dent Res 2016;2(1):157-162

19 Bernard TN Jr, Seibert CE. Pedicle diameter determined by computed tomography. Its relevance to pedicle screw fixation in the lumbar spine. Spine 1992;17(6, Suppl):S160-S163

20 Grivas TB, Savvidou O, Binos S, et al. Morphometric characteristics of the thoracolumbar and lumbar vertebrae in the Greek population: a computed tomography-based study on 900 vertebrae-"Hellenic Spine Society (HSS) 2017 Award Winner". Scoliosis Spinal Disord 2019;14(1):2

21 Wolf A, Shoham M, Michael S, Moshe R. Morphometric study of the human lumbar spine for operation-workspace specifications. Spine 2001;26(22):2472-2477

22 Alam MM, Waqas M, Shallwani H, Javed G. Lumbar morphometry: a study of lumbar vertebrae from a Pakistani population using computed tomography scans. Asian Spine J 2014;8(4):421-426

23 Kang KS, Song KS, Lee JS, Yang JJ, Song IS. Comparison of radiographic and computed tomographic measurement of pedicle and vertebral body dimensions in Koreans: the ratio of pedicle transverse diameter to vertebral body transverse diameter. Eur Spine J 2011;20(3):414-421

24 Marasini RP, Gautam P, Sherchan B, et al. A morphometric study of lumbar spine pedicles in Nep-alese population. JCMS Nepal. 2014;10 (4):12-7.

25 Maaly MA, Saad A, Houlel ME. Morphological measurements of lumbar pedicles in Egyptian population using computerized tomography and cadaver direct caliber measurements. The Egyptian Journal of Radiology and Nuclear Medicine. 2010 Dec 1;41(4):475-81.

26 Rajput Y .Morphometric Study of Lumbar Pedicles in Indian Population. International Journal of current Medical and Applied sciences. 2016, 13(1),61-64.

27 Singel TC, Patel MM, Goghil DV. A study of width and height of lumbar pedicles in saurashtra region. J. Anat. Soc. India. 2004, 53; 4-9.

28 Chadha M, Balain B, Maini L, Dhaon BK. Pedicle morphology of the lower thoracic, lumbar, and S1 vertebrae: an Indian perspective. Spine. 2003;28:744-749.

29 Acharya S, Dorje T, Srivastava A. Lower dorsal and lumbar pedicle morphometry in Indian population: a study of four hundred fifty vertebrae. Spine (Phila Pa 1976).2010;35:E378-84.

30 Singh J ,Pahuja K, Khatri JK. Morphometeric analysis of the lumbar vertebrae in North Indian Population. Asian J Pharm Hea Sci 2013;3(4) 